\title{
Ridurre i problemi comportamentali nei bambini piccoli attraverso il video-feedback. Trial clinico randomizzato pragmatico
}

\author{
O'Farrelly $C$, Watt $H$, Babalis D, et al. \\ A Brief Home-Based Parenting Intervention to Reduce Behavior Problems in Young Children: A Pragmatic Randomized Clini- \\ cal Trial \\ JAMA Pediatr. 2021 Jun 1;175(6):567-576
}

\begin{abstract}
Viene studiato l'utilizzo della tecnica di videofeedback (Video-feedback Intervention to promote Positive Parenting and Sensitive Discipline - VIPP-SD) a domicilio. Ė il primo RCT di video-feedback condotto in un contesto di cure primarie. Sono state coinvolte 300 famiglie con bambini di età 12- 36 mesi che allo screening di reclutamento presentavano un punteggio nella fascia superiore per problemi di comportamento (Strengths and Difficulties Questionnaire -SDQ). L'intervento si è dimostrato efficace nel ridurre problemi comportamentali precoci in bambini di 1 e 2 anni. I benefici dell'intervento si notano infatti nei problemi di comportamento piuttosto che nei problemi di attenzione e sono più evidenti quando i sintomi iniziali sono peggiori. Non sono state invece registrate differenze apprezzabili per tutti gli esiti che riguardano i genitori (pratiche educative, umore, ansia, funzionamento della coppia genitoriale).
\end{abstract}

Reducing behavioural problems in young children through video feedback. A pragmatic randomized clinical trial

The use of videofeedback technique at home (Video-feedback Intervention to promote Positive Parenting and Sensitive Discipline - VIPP-SD) is evaluated. This is the first video-feedback RCT carried out in a primary care setting. 300 families with children aged 12-36 months who had an upper range score for behaviour problems (Strengths and Difficulties Questionnaire - SDQ) were involved. The intervention proved to be effective in reducing early behavioural problems in 1 and 2 year old children. The benefits of the intervention are seen in behavioural problems rather than in attention problems and are more evident when the initial symptoms are worse. No appreciable differences were recorded for all the outcomes concerning parents (educational practices, mood, anxiety, functioning of the parental couple).

\section{Metodo}

\section{Obiettivo (con tipo studio)}

RCT, in singolo cieco, pragmatico, svolto in un contesto di cure primarie caratterizzato da un intervento domiciliare breve per ridurre i problemi comportamentali esternalizzanti nei bambini da 12 a 36 mesi attraverso l'utilizzo di una tecnica di videofeedback (Video-feedback Intervention to promote Positive Parenting and Sensitive Discipline - VIPP-SD) (Box 1) che ha lo scopo di promuovere una relazione positiva tra genitore e bambino e uno stile genitoriale responsivo.

\section{Popolazione}

Bambini di famiglie afferenti a 6 agenzie del servizio sanitario nazionale inglese, con un punteggio al Strengths and Difficulties Questionnaire (SDQ) (Box 2) che si colloca al quinto superiore. Criteri di inclusione:

- score elevato $(\geq 8)$ nella sottoscala dei disturbi esternalizzanti, allo SDQ;

- genitori/caregivers maggiorenni;

- rilascio di consenso informato scritto;

- bambini di età compresa tra 12 e 36 mesi.

Criteri di esclusione:

- bambini o genitori con deficit sensoriale, disturbi dell'apprendimento o del linguaggio;

- fratelli già reclutati nello studio;

- famiglia partecipante a studi analoghi che comprendevano interventi di video feedback;

- famiglie sottoposte a provvedimenti giudiziari.

\section{Intervento}

151 famiglie a cui sono state offerte 6 sedute domiciliari di VIPP-SD ( 4 sedute base e 2 di booster della durata di 1-2 ore ogni 2 settimane) con un professionista sanitario addestrato (prevalentemente infermieri o personale con formazione psicologica).

\section{Controllo}

149 famiglie a cui sono stati proposti solo gli interventi standard, poiché all'interno del NHS non esiste ancora un percorso codificato per i disturbi comportamentali ad esordio precoce.

\section{Outcome/Esiti}

Outcome primario: gravità dei disturbi del comportamento, valutata tramite l'uso di una versione del Parental Account of Children's Symptoms modificata per la prima infanzia (Preschool Parental Account of Children's Symptoms -PPACS).

Outcome secondari:

- score PPACS a distanza di 2 anni dalla randomizzazione (saranno riportati successivamente);

- disturbi del comportamento riferiti dai caregivers attraverso la compilazione di SDQ e del Child Behavior Checklist (CBCL);

- valutazione pratiche educative, umore dei genitori, loro apprensione e funzionamento della coppia genitoriale. 


\section{Tempo}

L'arruolamento dei pazienti è avvenuto tra il 30/07/2015 e il 26/07/2017.

La valutazione post-trattamento (follow-up) si è svolta tra il 9/12/2015 e il 27/04/2018.

L'analisi statistica è stata condotta tra il 5/09/2019 e il 17/01/2020 .

\section{Risultati principali}

2.248 potenziali partecipanti analizzati tra il 30 luglio 2015 e il 26 luglio 2017; 300 randomizzati (54\% maschi) di età media 23 mesi su 818 eleggibili. Sono disponibili i dati relativi all' outcome primario di 140 (su 151) partecipanti all'intervento di video-feedback, pari al 93\% (11 persi al follow up di cui 2 emigrati allestero) e di 146 (su 149) partecipanti agli interventi standard, pari al 98\% (3 persi al follow-up di cui 1 emigrato all'estero).

Outcome primario: differenza media di 2.03 (IC 95\% 0.06, 4.01; $\mathrm{p}=0.04, d$ di Cohen 0.20) nel punteggio del Preschool Parental Account of Children's Symptoms; minori problemi di comportamento nel gruppo video-feedback, in particolare riguardo ai sintomi relativi alla condotta: differenza media di 1.61 (IC 95\% $0.44,2.78 ; \mathrm{p}=0.007, d 0.30)$. Non sono stati rilevati effetti sull'iperattività ( $\mathrm{p} 0.67 ; d 0.05)$.

Gli outcome secondari mostrano evidenze simili a favore del video-feedback sui disturbi del comportamento del bambino, mentre non vi sono differenze apprezzabili per tutti gli altri esiti che riguardano i genitori (pratiche educative, umore, ansia, funzionamento della coppia genitoriale). Non sono stati riportati eventi avversi durante lo studio.

\section{Conclusioni}

L'intervento breve domiciliare di video-feedback si è dimostrato efficace nel ridurre i sintomi di problemi comportamentali precoci in bambini di 1 e 2 anni, quando viene effettuato nel contesto di un servizio di cure primarie. L'analisi dell'aderenza al trattamento evidenzia che chi riceve almeno 4 interventi di video-feedback ottiene miglioramenti più consistenti. I benefici dell'intervento si notano nei problemi di comportamento piuttosto che nei problemi di attenzione e sono più evidenti quando $i$ sintomi iniziali sono peggiori.

\section{Altri studi sull'argomento}

I due studi presentati in questa scheda newsletter e nella scheda "Migliorare l'interazione genitore-figlio dalla nascita tamite il video feed-back: uno studio randomizzato controllato" sempre in questo numero delle Pagine elettroniche di Quaderni acp indicano come un intervento tramite video feedback per sostenere efficacemente il parenting possa essere proposto in un contesto di cure primarie pediatriche.

Poiché sono stati svolti numerosi trial per sostenere il parenting, abbiamo rivolto l'attenzione alle revisioni sistematiche che analizzano gli effetti di interventi di sostegno del parenting all'interno delle cure primarie pediatriche e alle revisioni sistematiche di specifici interventi di video feedback per migliorare la responsività dei genitori.
Una revisione del 2016 ha raccolto 13 trial svolti nelle cure primarie rivolti a promuovere un parenting positivo in famiglie con bambini di età inferiore ai 3 anni; le metanalisi hanno rilevato un effetto positivo sull'interazione genitore-figlio (differenza media standardizzata DMS 0.29, IC $95 \% 0.06,0.52$ ) e sulle attività di stimolazione cognitiva (DMS 0.34, IC 95\% 0.03, 0.54); questi effetti positivi, anche se modesti, erano inficiati da una importante eterogeneità degli studi [1]. Una revisione sistematica di 48 studi (24 interventi) realizzati nelle cure primarie pediatriche con l'obiettivo di migliorare lo sviluppo del bambino ha rilevato 2 interventi efficaci nella riduzione del ritardo di sviluppo, 4 efficaci nell'aumentare lo sviluppo cognitivo e 6 che riducevano i problemi di comportamento. La estrema eterogeneità degli interventi (misure di outcome, follow-up, endpoint, tipologia di intervento) non ha permesso il confronto tra i diversi trial [2]. Una revisione sistematica pubblicata nel 2020 ha raccolto 40 studi svolti nelle cure primarie con intervento sui genitori ed esiti (cognitivi, emotivi e comportamentali) misurati sui bambini; nelle conclusioni gli autori sottolineano l'impatto positivo sulla salute mentale dei bambini e dei genitori della maggior parte degli interventi e indicano la necessità di strategie e di ricerca di implementazione di questi interventi nelle diverse realtà [3]. Infine, un'altra revisione sistematica pubblicata nel 2020 ha raccolto 17 studi (10 interventi) svolti nelle cure primarie pediatriche con intervento sulla funzione genitoriale ed esaminato come outcome il comportamento del bambino; gli autori hanno riscontrato risultati positivi nell'acquisizione delle conoscenze, nel luogo del controllo, nelle interazioni genitore-figlio e nella riduzione della disciplina negativa. Tuttavia gli esiti comportamentali sul bambino non sono apparsi univoci [4].

Nella ricerca di revisioni sistematiche specifiche dell'utilizzo del video feedback in età pediatrica abbiamo trovato 3 pubblicazioni. La prima revisione sistematica del 2018 raccoglie 29 studi pubblicati tra il 1990 e il 2014 riguardanti bambini fino a 12 anni di età. Gli effetti dei programmi di intervento tramite video feedback sono stati nel gruppo di intervento da moderati a grandi sulla sensibilità materna e sul comportamento dei bambini; tuttavia gli autori della revisione segnalano mancanza di chiarezza sulla tipologia specifica di intervento e una eterogeneità negli strumenti per misurare gli esiti [5].

Una revisione Cochrane del 2019 ha raccolto 22 studi riguardanti bambini di età inferiore ai 5 anni (1.889 diadi o gruppi familiari); una metanalisi di 20 studi ha rilevato un miglioramento della sensibilità dei genitori rispetto ai controlli (differenza media standardizzata 0.34 IC $95 \% 0.20,0.49$ ) e una metanalisi su 2 studi ha rilevato un aumento della probabilità di attaccamento sicuro nel post-intervento (OR 3.04, IC 95\% 1.39, 6.67); tutte queste misurazioni sono state rilevate tramite la Strange Situation. 8 interventi hanno misurato eventuali effetti avversi senza rilevare differenze tra gruppo di intervento e gruppo di controllo. Gli autori della revisione nelle conclusioni affermano la presenza di prove di moderata certezza che il video feedback può migliorare la sensibilità dei genitori nei bambini con difficoltà di attaccamento [6]. Una metanalisi a rete del 2021 ha incluso 119 studi confrontando 16 differenti combinazioni di intervento con il comune obiettivo di migliorare la responsività del genitore. L'effetto complessivo dei programmi analizzati è stato di grado medio ( $d$ di Cohen 0.56 IC 95\% 0.47, 0.65); i programmi più efficaci includevano l'insegnamento didattico, le opportunità per i ge- 
nitori di osservare modelli, mettere in pratica le abilità apprese, il feedback e il riflettere sulla propria esperienza di responsività. Inoltre i programmi più efficaci erano quelli dove: a) i genitori hanno osservato esempi di responsività, b) erano presenti i ricercatori come facilitatori, c) erano assegnati compiti a domicilio, d) il focus di intervento era ristretto. Nelle conclusioni gli autori della metanalisi a rete indicano che le home visit e i video-feedback personalizzati sono interventi efficaci per aumentare la responsività del genitore in situazione di rischio e che interventi brevi focalizzati su uno specifico obiettivo possono essere più efficaci di programmi di intervento prolungati e più costosi [7].

\section{Che cosa aggiunge questo studio}

È il primo RCT di video-feedback condotto in un contesto di cure primarie, che non necessita di un setting speciale e che ha dimostrato efficacia in bambini molto piccoli, in un'ottica di prevenzione di problemi di salute mentale.

\section{Commento}

\section{Validità interna}

Disegno dello studio: studio di elevata qualità metodologica. Lo studio è stato realizzato secondo le LG CONSORT. Il protocollo di studio è registrato. Gli autori riportano una variazione del protocollo accettabile (follow-up a 5 mesi invece che a 4 come inizialmente stabilito, per mancato completamento dell'intervento). Degli eleggibili più di $1 / 3$ partecipa allo studio. La randomizzazione, che viene descritta accuratamente, è adeguata. Come spesso accade per questi studi il livello educativo dei genitori era superiore a quello medio della popolazione generale. La cecità, singola, è adeguata per il tipo di studio. La fidelity, sia degli intervistatori che di chi ha effettuato l'intervento di videofeedback, è stata monitorata e valutata a campione (10\%). L'aderenza al trattamento è stata alta: 1'80\% ha svolto tutte e 6 le sessioni previste e l'85\% almeno 4 . Risultati più significativi sono associati all'avere effettuato almeno 4 sessioni. Solo 12 soggetti nel gruppo intervento non hanno effettuato alcuna sessione. I persi al follow-up sono stati pochi: 11/151 nel gruppo intervento e 3/149 nei controlli. Gli eventi avversi nel periodo di follow-up sono pochi, bilanciati nei due gruppi e non correlabili all'intervento. L'analisi dei risultati è stata fatta per intention to treat. Sono stati valutati diversi scenari per la gestione dei dati mancanti e anche l'analisi che considera lo scenario peggiore conferma la validità dell'intervento anche se l'intervallo di confidenza per pochissimo non è significativo.

Esiti: rilevanti e clinicamente definiti. Anche se è mancata una valutazione indipendente dal genitore, l'intervista PPACS permettere di misurare severità e frequenza dei comportamenti esternalizzanti e la codifica avviene da parte dell'operatore e non tramite il giudizio del genitore.

Conflitto di interesse: lo studio è stato finanziato dal National Institute for Health Research (NIHR) Health Technology Assessment (HTA) e dal NIHR Biomediacal Research Centre based at Imperial College London. Questi enti non hanno avuto un ruolo nel disegno dello studio, la raccolta dei dati, la loro interpretazione o nella stesura dell'articolo. I Dr. Bakermans-Kranenburg e van IJzendoorn hanno sviluppato il VIPP-SD.

\section{Trasferibilità}

Popolazione studiata: la popolazione studiata è sovrapponibile a quella che accede ai nostri studi. Spesso a partire dal secondo anno di vita, in una fase di sviluppo fisiologicamente oppositiva, i genitori portano al pediatra difficoltà comportamentali che si presentano nel contesto famigliare e/o scolastico e hanno la necessità di essere sostenuti nell'attuare una disciplina positiva. Tipo di intervento: attualmente questo tipo di intervento non è in uso in Italia su larga scala e in modo strutturato all'interno del sistema sanitario pubblico né nelle cure primarie né nei servizi di NPI. È possibile effettuare il training formativo presso il Laboratorio di Psicologia dell'Attaccamento e Sostegno alla Genitorialità - LAG Università degli Studi di Pavia. Ci sono alcuni terapisti formati che lavorano in diverse regioni d'Italia. I progetti a sostegno della genitorialità che prevedono interventi domiciliari sono attualmente poco diffusi all'interno delle cure primarie. L'utilizzo di un intervento come il VIPP-SD da realizzare in un contesto ambulatoriale e all'interno di un percorso condiviso con i servizi di NPI per la gestione dei problemi comportamentali a partire dai primi anni di vita, potrebbe essere utile anche per la nostra realtà.

1. Shah R, Kennedy S, Clark MD, et al. Primary care-based interventions to promote positive parenting behaviors: A meta-analysis. Pediatrics. 2016;137(5):e20153393. doi: 10.1542/peds.2015-3393.

2. Peacock-Chambers E, Ivy K, Bair-Merritt M. Primary Care Interventions for Early Childhood Development: A Systematic Review. Pediatrics. 2017;140(6):e20171661. doi: 10.1542/peds.2017-1661.

3. Smith JD, Cruden GH, Rojas LM, et al. Parenting Interventions in Pediatric Primary Care: A Systematic Review. Pediatrics. 2020;146(1):e20193548. doi: 10.1542/peds.2019-3548.

4. Moon DJ, Damman JL, Romero A. The Effects of Primary Care-Based Parenting Interventions on Parenting and Child Behavioral Outcomes: A Systematic Review. Trauma Violence Abuse. 2020;21(4):706-724. doi: 10.1177/1524838018774424. Epub 2018 Jul 31.

5. Balldin S, Fisher PA, Wirtberg I. Video feedback intervention with children: a systematic review. Research on Social Work Practice. 2018;28(6):682-95.

6. O'Hara L, Smith ER, Barlow J, et al. Video feedback for parental sensitivity and attachment security in children under five years. Cochrane Database of Systematic Reviews. 2019(11).

7. Sokolovic N, Rodrigues M, Tricco AC, et al. Teaching Parents to Be Responsive: A Network Meta-analysis. Pediatrics. 2021;148(2):e2020033563. doi: 10.1542/peds.2020-033563.

\section{Scheda redatta dal gruppo di lettura di Reggio Emilia:}

Francesca Bontempo, Carlo Boni, Jennifer Chiarolanza, Angelo Cigarini, Elena Corbelli, Annalisa Correggi, Anna Maria Davoli, Anna Rita Di Buono, Elena Ferrari, Monica Malventano, Elena Manzotti, Maddalena Marchesi, Gino Montagna, Luciana Monti, Rosaria Ollari, Luisa Seletti, Mariassunta Torricelli, Maria Candida Tripodi, Daniela Vignali, Marialuisa Villani, Costantino Panza. 


\section{Box 1}

\section{Video-feedback Intervention to promote Positive Parenting and Sensitive Discipline}

Il Video-feedback Intervention to Promote Positive Parenting and Sensitive Discipline (VIPP-SD) è un breve intervento evidence-based di sostegno alla genitorialità, ispirato alla teoria dell'attaccamento e articolato in 6 incontri domiciliari, volti a migliorare e sostenere le capacità di comunicare ed educare, in maniera efficace, il proprio bambino da parte del genitore. L'intervento è finalizzato a promuovere la disciplina sensibile nel genitore e a sviluppare una miglior comunicazione emotiva fra genitore e bambino. Mira ad accrescere la sensibilità genitoriale e la capacità di utilizzare strategie disciplinari sensibili ed efficaci e a prevenire lo sviluppo di problematiche comportamentali. L'intervento si rivolge a famiglie con bambini di età tra i 18 mesi e i 6 anni. Nella sessione VIPP-SD, uno o entrambi i genitori guardano e riflettono con il terapeuta su un video precedentemente registrato durante una sessione di gioco tra genitore e figlio. Viene seguito un protocollo in sei step. Con lo svolgimento di una sessione, due tipi generali di attenzione terapeutica diventano possibili: uno è concentrandosi su ciò che viene chiamato il "filmato esterno", ovvero il comportamento visibile sia del genitore che del figlio. L'altro è sul "film interiore", cioè ciò che il genitore ha soggettivamente sperimentato durante l'interazione, e/o ciò che il bambino ha forse soggettivamente sperimentato (su quest'ultimo si riflette utilizzando tecniche di mentalizzazione). Una certa attenzione è sempre data al film esterno. A seconda delle circostanze (ad esempio, gli obiettivi della sessione, la disponibilità del genitore) anche il lavoro con il 'film interno' può essere portato avanti. I terapisti imparano anche una serie specifica di categorie, una "mappa di scansione", per guardare un video in preparazione di una sessione. Tipicamente nella sessione, nel passaggio 1 , il terapeuta mostra una parte selezionata del video e al paziente viene chiesto di commentare. Il paziente o i pazienti (ad esempio, una coppia di genitori) sono incoraggiati a condividere ciò che hanno trovato di interesse per il video. La discussione sulla base di queste osservazioni può proseguire. Nel passaggio 2, il terapeuta sottolinea una serie di momenti positivi visibili nell'interazione vista nel video, e condivide le sue valutazioni. Nel passaggio 3, il terapeuta si rivolge e offre un linguaggio diplomatico e non frontale per gli aspetti problematici nell'interazione. Molto spesso viene selezionato un solo problema. Nel passaggio 4, il pattern problematico appena evidenziato è esplorato. Nella fase 5 , il terapeuta e il paziente riflettono insieme su una o più nuove azioni che il paziente può fare a casa: l'attenzione si sposta ora al quando, dove, e come del cambiamento comportamentale. Nella fase 6, il terapeuta riassume i principali punti elaborati nella sessione.

\section{Box 1}

\section{Questionari utilizzati per la valutazione dei problemi di comportamento}

\section{Strengths and Difficulties Questionnaire}

25 domande in 5 sottoscale:

1. Scala dei problemi emotivi

2. Scala dei problemi di condotta

3. Scala dell'iperattività

4. Scala dei problemi con i pari

5. Scala dei comportamenti pro-sociali

Per ognuno dei 5 item delle sottoscale può essere attribuito un punteggio da 0 a 2

[non vero - parzialmente vero - assolutamente vero]

Gli item della sottoscala problemi di condotta sono:

- Spesso ha crisi di collera o è di cattivo umore

- Generalmente obbediente, esegue di solito le richieste dell'adulto (reverse score)

- Spesso litiga con gli altri bambini o li infastidisce di proposito

- Spesso litigioso con gli adulti

- Può essere dispettoso con gli altri

Per le prime 4 sottoscale (problemi emotivi, di condotta, iperattività, con i pari) un punteggio maggiore indica maggiori difficoltà, al contrario nella scala dei comportamenti prosociali un punteggio maggiore indica minori problemi.

Mentre per l'SDQ 4-17 anni il 10\% della popolazione generale inglese presenta valori alti o molto alti riflettendo la prevalenza del $10 \%$ dei disordini psichiatrici in questa popolazione, per i bambini inglesi di $2-4$ anni circa l' $80 \%$ della popolazione presenta valori medi, il $12 \%$ leggermente alti, il $4 \%$ alti e il $4 \%$ molto alti (considerando le prime 4 sottoscale).

\section{Preschool Parental Account of Children's Symptoms -PPACS}

Il PPACS consiste in un'intervista ai caregivers, condotta da un esaminatore. Nel corso dell'intervista il caregiver primario deve descrivere il comportamento del bambino nel corso dell'ultima settimana specificando quanto questo sia rappresentativo degli ultimi 4 mesi. L'esaminatore giudica quindi la severità e la frequenza dei comportamenti valutando 2 sottogruppi di disturbi: disturbi della condotta e deficit di attenzione/ iperattività/ipercinesia. 